\title{
Intraoperative Radiation Therapy in Breast Cancer: Not Ready for Prime Time
}

\author{
Chirag Shah, MD ${ }^{1}$, Atif J. Khan, $\mathrm{MD}^{2}$, Douglas Arthur, $\mathrm{MD}^{3}$, Eduardo Fernandez, $\mathrm{MD}^{4}$, Alvaro A. Martinez, $\mathrm{MD}^{5}$, \\ and Frank Vicini, MD,FACR ${ }^{5}$ \\ ${ }^{1}$ Department of Radiation Oncology, Summa Health System, Akron, OH; ${ }^{2}$ Department of Radiation Oncology, Rutgers \\ Cancer Institute of New Jersey, New Brunswick, NJ; ${ }^{3}$ Department of Radiation Oncology, Virginia Commonwealth \\ University, Richmond, VA; ${ }^{4} 21$ st Century Oncology, Plantation, FL; ${ }^{5}$ Michigan Healthcare Professionals/21st Century \\ Oncology, Farmington Hills, MI
}

Breast conserving therapy remains one of the most important advances in cancer management in the past century, with multiple trials demonstrating its equivalence to mastectomy with respect to long term cancer outcomes. ${ }^{1,2}$ Unfortunately, studies have documented that adjuvant radiation therapy, a critical component of breast conserving therapy, remains under utilized. Data suggest that treatment duration (5-6.5 weeks) can frequently be attributed to this finding. ${ }^{3}$ In light of this and secondary to the increasing costs associated with breast cancer radiation therapy, shortened courses of radiation therapy have been explored including both hypofractionated whole breast irradiation (WBI) and accelerated partial breast irradiation (APBI) that shorten treatment duration to three and one week, respectively. Prospective randomized trials evaluating these techniques have been promising, demonstrating equivalence to traditional WBI with respect to clinical outcomes, toxicities, and breast cosmesis. ${ }^{4,5}$

Intraoperative radiation therapy (IORT) represents an alternative to these techniques that delivers treatment at the time of surgery as a single fraction of radiation (in the majority of cases). Increasingly, IORT is being offered to women with early-stage breast cancer as the definitive radiation therapy modality following breast conserving surgery. What is concerning is that these patients are being offered this, as yet, unproven therapy off-protocol with

(C) Society of Surgical Oncology 2013

First Received: 2 September 2013;

Published Online: 2 October 2013

C. Shah, MD

e-mail: csshah27@hotmail.com insufficient data to support its safety and efficacy compared with WBI or alternative techniques (APBI or hypofractionated WBI). This is unfortunate as radiation therapy in breast cancer represents an area of research that has undergone progress in the past five decades through systematic evidence-based treatment paradigm evolution; breast conserving therapy was verified via multiple randomized trials demonstrating comparable outcomes to mastectomy, and postmastectomy radiation therapy similarly demonstrated a survival benefit in several trials before wide-scale incorporation into treatment paradigms. ${ }^{1,2,6}$ Therefore, the rapid introduction of IORT is concerning as these steps have not been taken prior to wide-scale utilization, leading to a potential for higher rates of local recurrence or increased toxicity.

It should be noted that two large randomized trials have been performed to evaluate the role of IORT in early-stage breast cancer compared with WBI, the current standard of care. The targeted intraoperative radiotherapy (TARGIT) trial was a randomized noninferiority trial that included 3,451 women enrolled in 10 countries between 2000 and 2012. Patients were randomized to IORT (delivered at the same time as surgery or as a separate procedure) or WBI; however, $21 \%$ of the prepathology IORT patients received WBI because of predefined factors including lobular carcinoma in situ, extensive intraductal component, lymphovascular space invasion, node positivity, or other factors specific to each institution. IORT was delivered using a 50-kv X-ray source to deliver 20 Gy to the surface of the excision cavity $(5 \mathrm{~Gy}$ at $1 \mathrm{~cm}$ ). While initial data with short follow-up (median follow-up $<2$ years) and limited events $(n=11)$ was promising, a recent update found that IORT, even with WBI supplementation in one-fifth of the 
IORT cohort, was associated with inferior local control at 5 years (3.3 vs. $1.3 \%, p=0.04)^{7,8}$

A second randomized trial from Milan randomized 1,186 patients to IORT or WBI. This trial used IORT delivered with electrons (21 Gy delivered to the $90 \%$ isodose line). Importantly, WBI was not offered to any patients in the IORT cohort. With 6 years of follow-up, IORT was associated with an increased rate of local recurrences (5.3 vs. $0.7 \%, p<0.001)$ and true recurrences (3.2 vs. $0.7 \%, p=0.0002$ ) as well. ${ }^{9}$ Based on two large randomized trials showing an increase in local recurrence, it is inconceivable that IORT should be offered off-protocol, even in patients with low-risk disease.

Beyond clinical data, there are significant concerns regarding the radiobiology and physics regarding current IORT techniques. When examining the IORT using a $50-\mathrm{kv}$ X-ray source, it should recognized that while the dose at the surface is $20 \mathrm{~Gy}$, at $1 \mathrm{~cm}$ the dose is roughly only 5 Gy as a single dose; contrast this with a typical APBI plan that delivers $3.4 \mathrm{~Gy}$ at $1 \mathrm{~cm}$ over 10 fractions and it is not surprising that higher rates of local recurrence were noted in randomized trials using $50-\mathrm{kv}$ X-rays. ${ }^{10}$ In contrast, the electron (ELIOT) technique delivers 21 Gy to the $90 \%$ isodose line, a larger dose to the cavity than offered by the $50-\mathrm{kv}$ technique or APBI, raising concerns regarding treatment toxicity that have already been noted with regard to fat necrosis. ${ }^{9}$ Also, in light of the electron IORT trial omitting WBI regardless of pathological factors (surgical margin, nodal status, etc.), it is unlikely that this technique provides appropriate dose for those requiring regional nodal irradiation or more extensive breast volumes treated. Another major limitation of IORT is a lack of image guidance; there is an inability to identify and document where dose is delivered with respect to the excision cavity as well as the organs at risk including the skin and chest wall. This limits the ability to document the quality of treatment or in cases where re-treatment is needed to identify previously delivered dose to organs at risk.

Extrapolation from data supporting APBI is often used as a justification for the use of IORT. However, it should be noted that this extrapolation is not based on progressive logic. APBI initially defined its target and dose delivery based on in-breast failure patterns, pathologic studies, radiobiologic dose calculations and known brachytherapy dose delivery techniques with image guidance confirming dose delivery. Multiple successful single institutional studies and a large registry study set the stage for many phase III studies that are only now beginning to reach a maturity level where outcome can be determined and several societies have now defined a conservative group of patients that are identified as appropriate for off-protocol treatment with APBI. The selection criteria outlined is based on a large group of women treated with similar treatment designed based on the basic principles of target delineation and dose delivery upon which APBI was first established; IORT is a clear departure from those of APBI in both target definition and delineation as well as prescription dose and dose delivery. Although IORT may prove to have some advantages, it is illogical to extrapolate the APBI experience for off protocol use of IORT. If those promoting IORT abandon the pursuit of continued on-study use in exchange for commercial benefit of rapid introduction into widespread use, there is risk that assumptions upon which the use of this technique are founded are wrong and the potential benefit of this treatment approach will be lost. Finally, it should be noted that recent studies have demonstrated the feasibility of delivering APBI over 2 days with a dose based on whole breast and partial breast studies. Future studies will be needed to further evaluate this, offering patients a shortened course based on much stronger data than that currently available supporting IORT. ${ }^{11}$

In light of increasing costs associated with radiation therapy delivery, advocates of IORT have cited a potential in cost savings associated with the implementation of IORT. ${ }^{12,13}$ However, it should be noted that while IORT may represent a cost savings when using absolute costs, when factoring in the higher rates of local recurrence noted in randomized trials and the costs associated with treatment of these recurrences, the initial bargain that IORT represent may fade away.

In summary, IORT represents a promising area of investigation but should not be considered a standard of care in the management of early-stage breast cancer in light of (1) clinical data from randomized trials documenting inferior outcomes to WBI, (2) a failure to adhere to standard radiobiological principles used in breast cancer, and (3) a lack of standardized protocols for delivery. Moving forward, we support the continued study of the concept but feel that it should remain as a technique under investigation and not advertised as a new standard in the management of early-stage breast cancer or used off-protocol until clinical data support it.

\section{CONFLICT OF INTEREST None.}

FUNDING None.

\section{REFERENCES}

1. Fisher B, Anderson S, Bryant J, Margolese RG, Deutsch M, Fisher ER, et al. Twenty-year follow-up of a randomized trial comparing total mastectomy, lumpectomy, and lumpectomy plus irradiation for the treatment of invasive breast cancer. $N$ Engl J Med. 2002;347:1233-41.

2. Van Dongen JA, Voogd AC, Fentiman IS, Legrand C, Sylvester $\mathrm{RJ}$, Tong D, et al. Long-term results of a randomized trial comparing breast-conserving therapy with mastectomy: European 
Organization for Research and Treatment of Cancer 10801 trial. $J$ Natl Cancer Inst. 2000;92:1143-50.

3. Feinstein AJ, Soulos PR, Long JB, Herrin J, Roberts KB, Yu JB, et al. Variation in receipt of radiation therapy after breast-conserving surgery: assessing the impact of physicians and geographic regions. Med Care. 2013;51:3308-8.

4. Whelan TJ, Pignol JP, Levine MN, Julian JA, MacKenzie R, Parpia S, et al. Long-term results of hypofractionated radiation therapy for breast cancer. N Engl J Med. 2010;362:513-20.

5. Polgar C, Fodor J, Major T, Major T, Sulyok Z, Kasler M. Breastconserving treatment with partial or whole breast irradiation: 10 year results of the Budapest randomized trials. Radiother Oncol. 2013. doi:10.1016/j.radonc.2013.05.008.

6. Ragaz J, Olivotto IA, Spinelli JJ, Phillips N, Jackson SM, Wilson $\mathrm{KS}$, et al. Locoregional radiation therapy in patients with highrisk breast cancer receiving adjuvant chemotherapy: 20-year results of the British Columbia randomized trial. J Natl Cancer Inst. 2005;97:116-26.

7. Vaidya JS, Joseph DJ, Tobias JS, Bulsara M, Wenz F, Saunders $\mathrm{C}$, et al. Targeted intraoperative radiotherapy versus whole breast radiotherapy for breast cancer (TARGIT-A trial): an international, prospective, randomised, non-inferiority phase 3 trial. Lancet. 2010;376:91-102.

8. Vaidya JS, Wenz F, Bulsara M, Joseph D, Tobias JS, Keshtgar M, et al. Targeted intraoperative radiotherapy for early breast cancer:
TARGIT-A trial—updated analysis of local recurrence and first analysis of survival. http://www.abstracts2view.com/sabcs12/view. php?nu=SABCS12L_3020\&terms=. Accessed 24 Aug 2013.

9. Orecchia R. IORT update: what are the limits of radiation dose and volume? http://webcast.streamdis.eu/mediasite/Catalog/pages/catalog. aspx?catalogId=5713e723-b087-4ac0-9098-e9da8ab27fcf. Accessed 24 Aug 2013.

10. Khan AJ, Arthur DW, Vicini FA. On the road to intraoperative radiotherapy: more proceed with caution signs. Oncology. 2013;27:113-4.

11. Wilkinson JB, Martinez AA, Chen PY, Ghilezan MI, Wallace MF, Grills IS, et al. Four-year results using balloon-based brachytherapy to deliver accelerated partial breast irradiation with a 2-day dose fractionation schedule. Brachytherapy. 2012;11: 97-104.

12. Alvarado MD, Mohan AJ, Esserman LJ, Park CC, Harrison BL, Howe RJ, et al. Cost-effectiveness of intraoperative radiation therapy for early-stage breast cancer. Ann Surg Oncol. 2013; 20:2873-80.

13. Grobmyer SR, Lightsey JL, Bryant CM, Shaw C, Yeung A, Bhandare $\mathrm{N}$, et al. Low-kilovoltage, single-dose intraoperative radiation therapy for breast cancer: results and impact on a multidisciplinary breast cancer program. $J$ Am Coll Surg. 2013;216:617-23, discussion 623-4. 